\title{
Clinical Outcomes and Return to Sports in Patients with Chronic Achilles Tendon Rupture after Minimally Invasive Reconstruction with Semitendinosus Tendon Graft Transfer
}

\author{
Federico Giuseppe Usuelli ${ }^{1}$ Riccardo D’Ambrosi \\ Jorge Hugo Villafañe ${ }^{3}$ Pedro Berjano ${ }^{1}$ \\ ${ }^{1}$ IRCCS Istituto Ortopedico Galeazzi, Milan, Italy \\ 2 Dipartimento di Scienze Biomediche per la Salute, IRCCS Istituto \\ Ortopedico Galeazzi, Università degli Studi di Milano, Milan, Italy \\ 3 IRCCS Fondazione Don Carlo Gnocchi, Milan, Italy
}

\author{
Luigi Manzi $^{1} \quad$ Cristian Indino $^{1}$
}

Joints 2017;5:212-216.

\begin{abstract}
Address for correspondence Riccardo D’Ambrosi, MD, Università degli Studi di Milano, Dipartimento di Scienze Biomediche per la Salute, IRCCS Istituto Ortopedico Galeazzi, Via Galeazzi 4, Milan 20161, Italy (e-mail: riccardo.dambrosi@hotmail.it).
\end{abstract}

\begin{abstract}
Keywords

- chronic Achilles tendon rupture

- tendon transfer

- return to sport

Objective The purpose of the study is to evaluate the clinical results and return to sports in patients undergoing reconstruction of the Achilles tendon after minimally invasive reconstruction with semitendinosus tendon graft transfer.

Methods Eight patients underwent surgical reconstruction with a minimally invasive technique and tendon graft augmentation with ipsilateral semitendinosus tendon for chronic Achilles tendon rupture (more than 30 days after the injury and a gap of $>6 \mathrm{~cm}$ ). Patients were evaluated at a minimum follow-up of 24 months after the surgery through the American Orthopaedic Foot and Ankle Society (AOFAS), the Achilles Tendon Total Rupture Scores (ATRS), the Endurance test, the calf circumference of the operated limb, and the contralateral and the eventual return to sports activity performed before the trauma.

Results The mean age at surgery was 50.5 years. Five men and three women underwent the surgery. The average AOFAS was 92, mean Endurance test was 28.1, and the average ATRS was 87 . All patients returned to their daily activities, and six out of eight patients have returned to sports activities prior to the accident (two football players, three runners, one tennis player) at a mean of 7.0 (range: 6.7-7.2) months after the surgery. No patient reported complications or reruptures.

Conclusion Our study confirms encouraging results for the treatment of Achilles tendon rupture with a minimally invasive technique with semitendinosus graft augmentation. The technique can be considered safe and allows patients to return to their sports activity.

Level of Evidence Level IV, therapeutic case series.
\end{abstract}

\section{Introduction}

Achilles tendon rupture occurs mainly in two categories of patients: young athletes and older patients ( $>50$ years) not related to sports. ${ }^{1-3}$ Chronic Achilles tendon ruptures are relatively uncommon, and are defined as a rupture of the Achilles tendon with an interval between rupture and treatment of at least weeks. ${ }^{4}$ In such patients, the triceps surae is shortened, and the gap between the proximal and distal stumps of the Achilles can be filled up with fibrous tissue. ${ }^{5,6}$ published online November 13, 2017
DOI https://doi.org/ $10.1055 / \mathrm{s}-0037-1608661$. ISSN 2282-4324.
Copyright @ 2017 Georg Thieme Verlag KG Stuttgart · New York
License terms

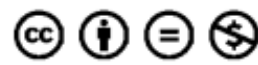


Plantar flexion of the ankle is reduced and residual movement occurs through the action of the secondary plantar flexors of the ankle joint. ${ }^{7}$ The management of this rupture presents a high risk of complications such as wound breakdown and infections. ${ }^{8}$ In ruptures with a large gap $(>6 \mathrm{~cm})$, the semitendinosus tendon can be used to undertake the reconstruction. ${ }^{9}$

The aim of this study is to assess clinical results and return to sports in eight patients with chronic rupture of the Achilles tendon and treated with the minimally invasive technique of reconstruction with semitendinosus tendon graft augmentation with a minimum follow-up of 24 months.

\section{Methods}

Eight consecutive patients were enrolled ( 5 men and 3 women; mean age 50.5) with chronic closed rupture of the Achilles tendon who had undergone surgical reconstruction with a minimally invasive technique and tendon graft augmentation with ipsilateral semitendinosus tendon.

All subjects gave their written informed consent to participate in the study, which was approved by the Ethical Review Committee.

Patients were enrolled if they had a diagnosis of a chronic closed rupture of the Achilles tendon on the basis of clinical and radiological examination. The average time between injury and surgical procedure was 40.4 days (range: 31-60). Both right and left sides of patients were affected. The patients were enrolled if magnetic resonance imaging (MRI) was available and showed a gap between the proximal and distal stumps of the Achilles tendon of at least $6 \mathrm{~cm}$ with the ankle in maximal plantar flexion. All patients also underwent an ultrasound evaluation to study the Achilles tendon. At clinical examination, all patients had a palpable gap at the site of rupture; furthermore, they presented a nonpropulsive gait on the affected limb and were not able to stand on their tiptoes.

The clinical diagnosis of Achilles tendon rupture was made using the Single Leg Heel Rise test, the Calf Squeeze test, and the Knee Flexion test. ${ }^{10}$

In the first test, the patient is asked to perform a single leg heel rise. If the patient is able to lift his heel against gravity, the Achilles tendon is intact. In the Calf Squeeze test (or Thompson's test), the patient lies prone with both feet hanging from the examination table. The examiner squeezes the affected calf muscle; if the Achilles tendon is intact, the foot will remain plantar flexed. If the Achilles tendon is torn, the foot will remain in the resting position, or only minimal plantar flexion will occur. On the affected side, the calf muscle should be squeezed at the level where the largest range of motion will be reached on the healthy side. In the last test, the patient lies prone, and is asked to flex both knees to $90^{\circ}$. During this movement, the position of both ankles is observed. An Achilles tendon rupture is diagnosed if the foot of the affected limb falls into neutral or dorsiflexion. If the tendon is intact, the foot will remain slightly plantar flexed. All assessments were performed by a clinician. Minimum follow-up was 24 months (mean 27.9; range 24-34).

\section{Surgical Procedure}

The patient is in prone position with high tourniquet. The first incision is performed $2 \mathrm{~cm}$ proximal and is just medial to the palpable end of the residual tendon. The second incision is $2 \mathrm{~cm}$ distal in the midline over the distal end of the tendon rupture. Care must be taken to avoid the sural nerve.

The two stumps of the tendon are then mobilized, releasing any possible adherence. The ruptured tendon end is then resected to the healthy tendon. The proximal tendon is then mobilized from the proximal wound, and is freed by adhesions, and further soft tissue release anterior to the soleus and gastrocnemius allows maximal excursion, minimizing the gap between the two stumps.

To harvest the semitendinosus, a vertical incision, 2.5 to $3 \mathrm{~cm}$ of length, is performed over the pes anserinus (-Fig. 1). The semitendinosus tendon is passed through a small incision in the substance of the proximal stump of the Achilles tendon using an absorbable suture (-Fig. 2). At this point, the semitendinosus is passed under the intact skin bridge to the distal incision, and passed from medial to lateral through a transverse tenotomy in the distal stump (-Fig. 3). Maintaining the ankle in maximal plantar flexion, the semitendinosus tendon is sutured to the Achilles tendon at each entry and exit point. Repair is tensioned to maximal equines. One extremity of the semitendinosus tendon is then passed again beneath the intact skin bridge into the proximal incision and passed from medial to lateral through a transverse tenotomy in the proximal stump. The same step is performed with the other extremity of the semitendinosus tendon with the distal stump of the Achilles tendon.

\section{Postoperative Rehabilitation}

Patients were discharged within 24 hours of the operation. The operated leg was positioned in a cast immediately after surgery, in neutral position $\left(90^{\circ}\right)$ and was held for approximately 40 days. No weight bearing was allowed until cast removal.

\section{Clinical Evaluation}

Clinical evaluation consisted of The American Orthopaedic Foot and Ankle Society (AOFAS) clinical rating system, the Achilles Tendon Total Rupture Score (ATRS), the Endurance test, the calf circumference of the operated limb, and the contralateral and the eventual return to sports activity

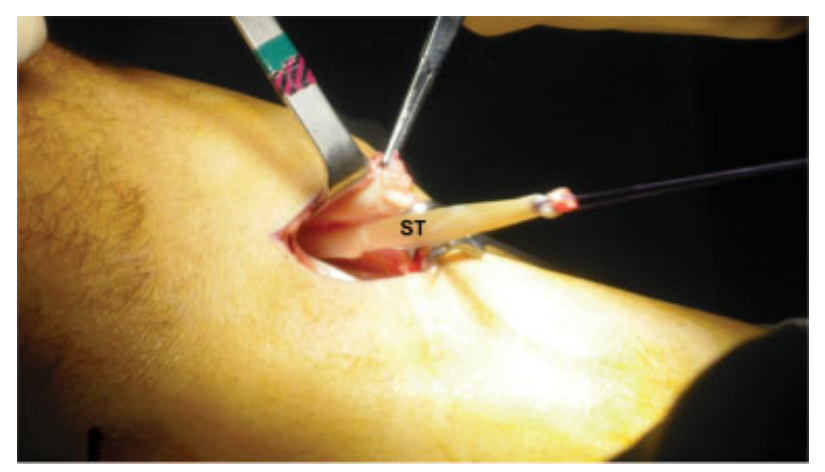

Fig. 1 Harvesting of the semitendinosus tendon through a vertical, $2.5-3 \mathrm{~cm}$ longitudinal incision over the pes anserinus.

ST, semitendinosus. 


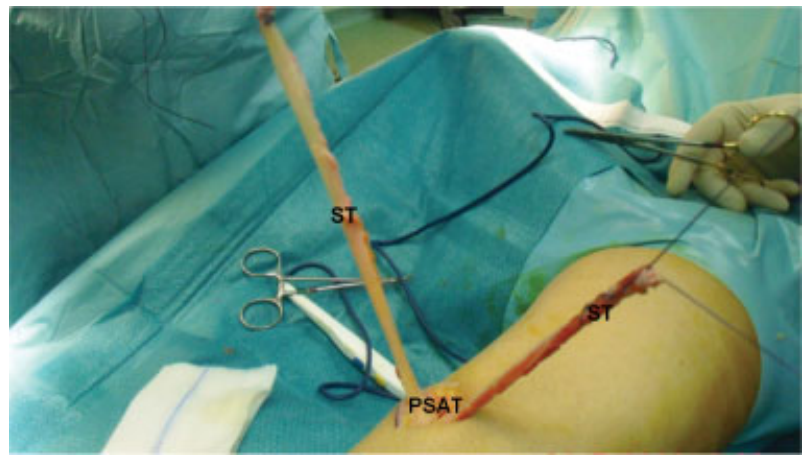

Fig. 2 With the patient prone, the semitendinosus is sutured to the proximal stump of the Achilles tendon. ST, semitendinosus; PSAT, proximal stump of the Achilles tendon.

performed before the trauma and time for return to sport. $^{11-14}$

The AOFAS ${ }^{11}$ has developed four rating systems in which the clinical status of the ankle and foot is reported. These systems incorporate both subjective and objective factors and are the most used scale in articles dealing with foot and ankle pathologies. ${ }^{15}$

The ATRS ${ }^{12}$ evaluates aspects of symptoms and physical activity. The questionnaire consists of 10 items, where the score for each item ranges between 0 and 10 on a Likert scale, with a maximum score of 100 .

The Endurance test ${ }^{14}$ is the most commonly used test for measuring muscular endurance of the calf musculature. In a heel rise test, the plantar flexion of the ankle is performed repetitively while standing until fatigued. The normal number of toe-raise repetitions on one leg is regarded to be $\sim 25$.

Circumference measurements are often used to determine gross muscular hypotrophy; however, such measurements cannot be used to determine muscle quality. Furthermore, the calf muscle circumference has a weak correlation to calf muscle strength and endurance after treatment for Achilles tendon rupture.

\section{Results}

All patients were reviewed at the average follow-up of 27.9 months (range: 24-34 months) from surgery; no patient was lost to follow-up. Clinical results are reported in - Table 1.

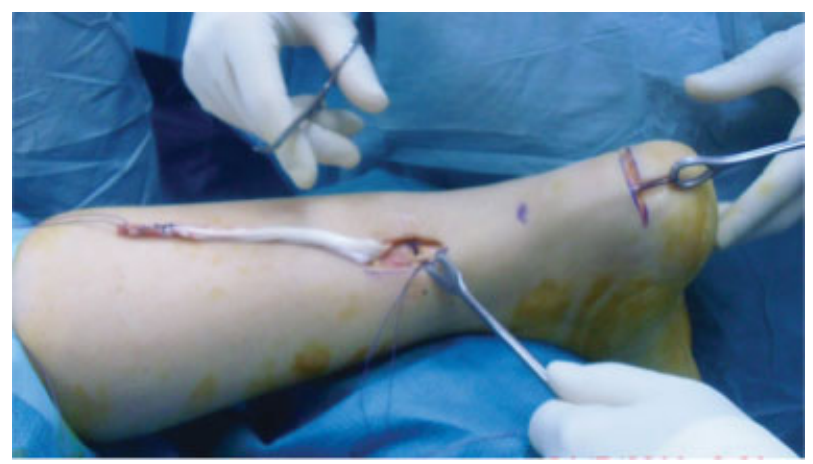

Fig. 3 The semitendinosus is passed under the skin bridge to the distal incision.
At the follow-up, the mean AOFAS was 92 (range: 83-96), mean ATRS was 87 (range: 81-95), mean Endurance test was 28.1 (range: $24-31$ ), and the mean calf circumference of the operated leg was $37.5 \mathrm{~cm}$ (range: $32.5-41.7$ ) in respect to the contralateral calf that was $38.5 \mathrm{~cm}$ (range: 33.0-42.5). All patients returned to their daily activities, and six out of eight patients have returned to sports activities ( 2 football players, 3 runners, 1 tennis player) at a mean of 7.0 (range: 6.77.2) months after surgery.

No patient reported complications either as regards to the symptoms related to the harvest of the semitendinosus or for the reconstruction of the Achilles tendon. There were no reported tendon reruptures of the tendon, issue of dehiscence of the surgical scar, or infections.

\section{Discussion}

Several techniques for Achilles tendon reconstruction have been described, including turndown flap, tendon transfer, tendon graft, or augmentation with synthetic materials. Tendon transfer is widely reported in literature with good results, but in some cases it can cause a loss of function. ${ }^{16}$ For the transfer of the flexor hallux longus, many patients have reported decrease in strength of the great toe flexor, whereas transfer of the flexor digitorum longus affects the push-off strength. ${ }^{17,18}$ For the peroneus brevis, its transfer can be associated with a decrease in eversion strength of the ankle. $^{19}$

In 2013, Maffulli et $\mathrm{al}^{20}$ treated 26 patients who had undergone minimally invasive semitendinosus autologous graft reconstruction for chronic ruptures to the Achilles tendon. At a mean follow-up of 8.2 years from surgery, the maximum calf circumference was significantly higher than preoperatively, and the mean ATRS was 88. Two patients developed a superficial wound infection, and one patient developed scar adhesion to the distal wound. All patients returned to their preinjury working occupation; 22 patients returned to their preinjury level of activity at a mean of 6.7 months after surgery.

Sarzaeem et $\mathrm{al}^{21}$ reported results in 11 consecutive patients, with a chronic Achilles tendon rupture with a gap of greater than $6 \mathrm{~cm}$ with the same technique. All patients have been evaluated at a mean follow-up of 25 months, and the average AOFAS and ATRS improved significantly from $70 \pm 5$ and $32 \pm 6$ preoperatively to $92 \pm 5$ and $89 \pm 4$ points postoperatively. The range of dorsiflexion was significantly limited on the operated side. All patients were able to stand on the tiptoe of the injured leg, and no patient walked with a visible limp. Postoperative complications included one patient with symptomatic deep vein thrombosis and two patients with superficial infection treated nonoperatively. The authors conclude that the technique offers good clinical and functional outcomes and is safe.

Dumbre et $\mathrm{al}^{22}$ treated 35 patients with symptomatic chronic Achilles tendon ruptures. The average follow-up duration was 30.7 months. Postoperatively, the strength of gastrocnemius and soleus was measured by manual muscle testing (MMT) in non-weight-bearing and weight-bearing positions. 


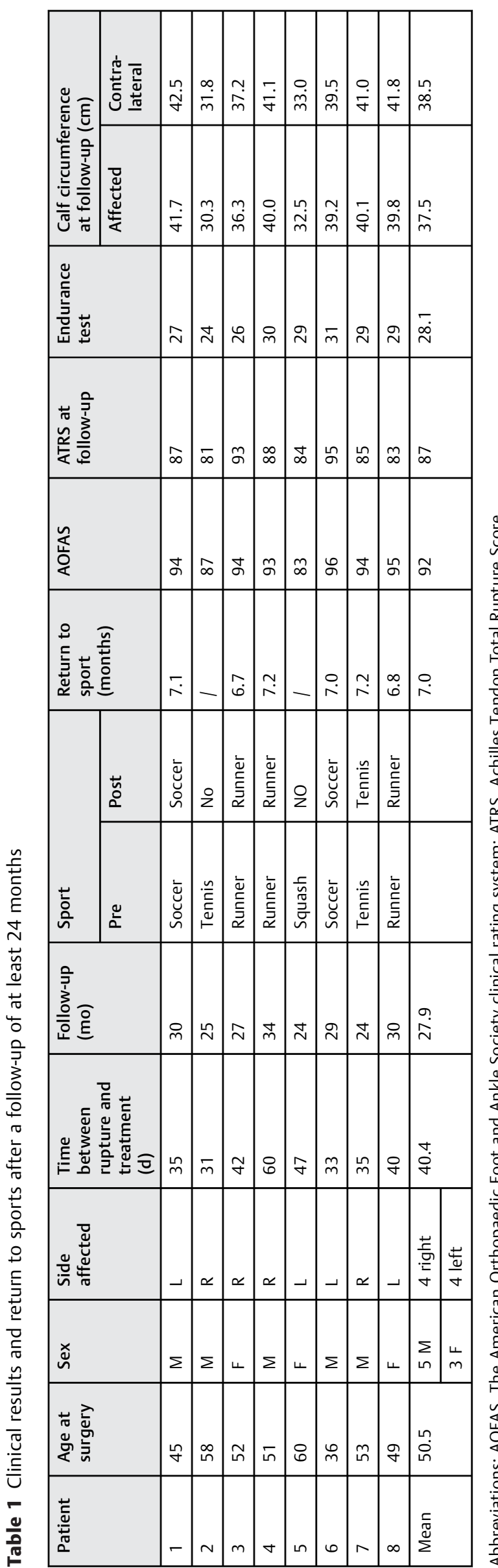

All operated patients showed satisfactory functional outcome, good soft tissue healing, and no reruptures. The preoperative weight-bearing MMT of $2 / 5$ improved to $4 / 5$ or $5 / 5$ postoperatively. In all patients, postoperative non-weight-bearing MMT was $5 / 5$. All patients returned to their prerupture daily activity. The authors believe that the technique is a valuable option especially when allografts are not available. It is inexpensive as suture anchors or tenodesis screws are not used. This can be a useful option if other tendons (flexor hallux longus, peroneus brevis, etc.) are not available for transfer.

Wound breakdown is challenging with complications at a rate of $9 \%$ in open technique, ${ }^{23}$ especially in chronic rupture of Achilles tendon. $3,19,24$

Minimally invasive technique allows reconstruction preserving skin integrity, reducing risk of scar infection or deihscence. ${ }^{25}$

Furthermore, the use of semitendinosus tendon can cover a gap of greater than $6 \mathrm{~cm}$. In fact semitendinosus is a long and strong tendon and provides a sturdy reconstruction of the Achilles tendon. ${ }^{19}$ The morbidity related to semitendinosus harvesting is minimal as it does not cause any loss of strength at the knee. ${ }^{7,26,27}$

To evaluate clinical benefits of the procedure, we used a well-validated scoring system.

The AOFAS has developed four rating systems, in which the clinical status of the ankle and foot is reported. The purpose of these rating systems was to have a clinically useful method, which could be used when comparing the results of different methods of treatment. These systems incorporate both subjective and objective factors.

The ATRS evaluates aspects of symptoms and physical activity. The questionnaire has good reliability, validity, and responsiveness for evaluating outcome after treatment in patients with tendon rupture. ${ }^{28}$

Limitation of the study is the lack of a control group and the small number of patients. An uncontrolled group design does not allow proving the superiority of this surgical technique for the management of chronic Achilles tendon rupture.

In conclusion, our study confirms, as already reported in literature, encouraging results for the treatment of Achilles tendon rupture with minimally invasive technique with semitendinosus graft augmentation. No complications have been reported and patients return to their sports activity.

Funding

None.

Conflict of Interest

Federico Giuseppe Usuelli, MD, reports personal fees from Integra and Geistlich, and grants and personal fees from Zimmer, outside the submitted work.

\section{References}

1 Ganestam A, Kallemose T, Troelsen A, Barfod KW. Increasing incidence of acute Achilles tendon rupture and a noticeable decline in surgical treatment from 1994 to 2013. A nationwide registry study of 33,160 patients. Knee Surg Sports Traumatol Arthrosc 2016;24(12):3730-3737 
2 Usuelli FG, Grassi M, Maccario C, et al. Intratendinous adiposederived stromal vascular fraction (SVF) injection provides a safe, efficacious treatment for Achilles tendinopathy: results of a randomized controlled clinical trial at a 6-month follow-up. Knee Surg Sports Traumatol Arthrosc 2017. Doi: 10.1007/s00167-017-4479-9

3 Maffulli N, Ajis A, Longo UG, Denaro V. Chronic rupture of tendo Achillis. Foot Ankle Clin 2007;12(04):583-596

4 Gabel S, Manoli A II. Neglected rupture of the Achilles tendon. Foot Ankle Int 1994;15(09):512-517

5 Maffulli N, Dymond NP, Capasso G. Ultrasonographic findings in subcutaneous rupture of Achilles tendon. J Sports Med Phys Fitness 1989;29(04):365-368

6 Myerson MS. Achilles tendon ruptures. Instr Course Lect 1999; 48:219-230

7 Padanilam TG. Chronic Achilles tendon ruptures. Foot Ankle Clin 2009;14(04):711-728

8 Longo UG, Ronga M, Maffulli N. Achilles tendinopathy. Sports Med Arthrosc Rev 2009;17(02):112-126

9 Maffulli N, Longo UG, Gougoulias N, Denaro V. Ipsilateral free semitendinosus tendon graft transfer for reconstruction of chronic tears of the Achilles tendon. BMC Musculoskelet Disord 2008;9:100

10 Maffulli N. The clinical diagnosis of subcutaneous tear of the Achilles tendon. A prospective study in 174 patients. Am J Sports Med 1998;26(02):266-270

11 Kitaoka HB, Alexander IJ, Adelaar RS, Nunley JA, Myerson MS, Sanders M. Clinical rating systems for the ankle-hindfoot, midfoot, hallux, and lesser toes. Foot Ankle Int 1994;15(07):349-353

12 Nilsson-Helander K, Thomeé R, Silbernagel KG, et al. The Achilles tendon Total Rupture Score (ATRS): development and validation. Am J Sports Med 2007;35(03):421-426

13 Paavola M, Kannus P, Orava S, Pasanen M, Järvinen M. Surgical treatment for chronic Achilles tendinopathy: a prospective seven month follow up study. Br J Sports Med 2002;36(03):178-182

14 Lunsford BR, Perry J. The standing heel-rise test for ankle plantar flexion: criterion for normal. Phys Ther 1995;75(08):694-698

15 Hunt KJ, Hurwit D. Use of patient-reported outcome measures in foot and ankle research.J Bone Joint Surg Am 2013;95(16):e118, 1-9

16 Longo UG, Lamberti A, Maffulli N, Denaro V. Tendon augmentation grafts: a systematic review. Br Med Bull 2010;94:165-188
17 Wapner KL, Pavlock GS, Hecht PJ, Naselli F, Walther R. Repair of chronic Achilles tendon rupture with flexor hallucis longus tendon transfer. Foot Ankle 1993;14(08):443-449

18 Jacob HA. Forces acting in the forefoot during normal gait-an estimate. Clin Biomech (Bristol, Avon) 2001;16(09):783-792

19 Carmont MR, Maffulli N. Less invasive Achilles tendon reconstruction. BMC Musculoskelet Disord 2007;8:100

20 Maffulli N, Del Buono A, Spiezia F, Maffulli GD, Longo UG, Denaro $\mathrm{V}$. Less-invasive semitendinosus tendon graft augmentation for the reconstruction of chronic tears of the Achilles tendon. Am J Sports Med 2013;41(04):865-871

21 Sarzaeem MM, Lemraski MM, Safdari F. Chronic Achilles tendon rupture reconstruction using a free semitendinosus tendon graft transfer. Knee Surg Sports Traumatol Arthrosc 2012;20(07): 1386-1391

22 Dumbre Patil SS, Dumbre Patil VS, Basa VR, Dombale AB. Semitendinosus tendon autograft for reconstruction of large defects in chronic Achilles tendon ruptures. Foot Ankle Int 2014;35(07): 699-705

23 Pintore E, Barra V, Pintore R, Maffulli N. Peroneus brevis tendon transfer in neglected tears of the Achilles tendon. J Trauma 2001; 50(01):71-78

24 Carmont MR, Maffulli N. Management of insertional Achilles tendinopathy through a Cincinnati incision. BMC Musculoskelet Disord 2007;8:82

25 Del Buono A, Volpin A, Maffulli N. Minimally invasive versus open surgery for acute Achilles tendon rupture: a systematic review. Br Med Bull 2014;109:45-54

26 Ardern CL, Webster KE, Taylor NF, Feller JA. Hamstring strength recovery after hamstring tendon harvest for anterior cruciate ligament reconstruction: a comparison between graft types. Arthroscopy 2010;26(04):462-469

27 Papandrea P, Vulpiani MC, Ferretti A, Conteduca F. Regeneration of the semitendinosus tendon harvested for anterior cruciate ligament reconstruction. Evaluation using ultrasonography. Am J Sports Med 2000;28(04):556-561

28 Kearney RS, Achten J, Lamb SE, Plant C, Costa ML. A systematic review of patient-reported outcome measures used to assess Achilles tendon rupture management: what's being used and should we be using it? Br J Sports Med 2012;46(16):1102-1109 